INNOVATIVE

JOURNAL

Contents lists available at www.innovativejournal.in INNOVATIVE JOURNAL OF MEDICAL AND HEALTH SCIENCE

Available online at

http://www.innovativejournal.in/index.php/ijmhs

ONLINE ISSN:2277-4939

PRINT ISSN:2589-9341

\title{
REVELATION OF ACUTE INTESTINAL INFEKTIONS' PERIODIC BIORYTHMS AND IDENTIFICATION OF DHE QUOLITATIVE PREVISIONS IN GYUMRI
}

\author{
ANDRYAN A.A.* \\ National Health, Information Analytic Center, Ministry of Health of the Republic of Armenia, Yerevan, \\ Armenia, and \\ National Center for Disease Control and Prevention of the Ministry of Health of the Republic of Armenia \\ SNCO "Shirak" Branch
}

\begin{abstract}
Longtime observations show that epidemical situation in Republic of Armenia has been and d continues to be difficult /problematicl, particularly in ters of intestinal infections. The natures of infectious deseases as well as their epidemiological patterns have been changed.

Revelation of the periodicity and the anpalysis of the peaks in frequency will help us to make possible previssions and insert goal-orientation into the epidemiological control.

Taking into consideration etiological, clinical and epidemiological features of intestinal infections during the constal analysis of intestinal infection deseases /1977-2011/ over the separate infections of intestinal group. We use the method of spectral analysis of the group of harmony and time /variation.

We have used the spectral analysis method of the time-series to clarify real epidemiological pattern and to make predictions, and with the help of Byui-Balo's hidden frequency's revelation method we have redused the probability of a possible error bringing the resulted frequency close to the real, natural biorythm.

Spectral analysisyears of time series, which characterize the avute intestinal infections, overoll morbidity of 34 years allows us to conform lestablish/ multirythmical characteristics of epidemiological dynamics. It turns out, that there is a specific periodicity for the deseases of intestinal group with separate nozological gorms.
\end{abstract}

Thus, theoretically, with the help of the above mentioned method, the following periodicity has been received for the total group of intestinal deseases with /- $(3,1+2,8)-(3,8+2,2)-5-4,6-2,2-5,1-2,1-2,3$ -year interval, which coincides with the practice activities with - 6 - 6 - 5 - 5 - 2 - 5 - 2 - 2 - regularity.

The investigated regularities predict approximate, probable periods /times/ of the risk. It is well known, that the periodic increase and decrease of the morbidity is different for the different germs of the infections of intestinal group. It also has different subspecies of the germ .

It should be noted that increases /peaks/ and falls have general tendency to decrease and stabilize for the all intestinal infections in terms of cumulative, namely there are on a lower level, comparate with the previous periodicity.

Biological cycle also depends on climatic factors associated with the improvement of conditions for development of the germ.

Using spectral analysis of time series, risk periods of intestinal separate infections were determined for Gyumri, which will be if not flashing, then probably years of high morbidity. According to previsions made by us, the frequency of separate infections has the following schedule:

Only permanent moniitoring and current and deep epidemiological research gives the opportunity to reveal distinct epidemiological regularities.

Computer mapping programs, especially Arc View GIS geographical informational system gives such an opportunity. With the help of digital maps resulted from this program, it is possible to get regional and allocation of infections, prevalence of indicators. it's also possible to determine the extent of contamination, which gives the opportunity to make epidemic measures intentional and effective to save means and resources.

Keywords: epidemiological freqency, intestinal/enteric/ infections, regularities, spectral analysis, quolitative prevision. 
ANDRYAN A.A. / REVELATION OF ACUTE INTESTINAL INFEKTIONS' PERIODIC BIORYTHMS AND IDENTIFICATION OF DHE QUOLITATIVE PREVISIONS IN GYUMRI

\section{Introduction:}

Longtime observations show that epidemical situation in Republic of Armenia has been and $d$ continues to be difficult /problematic/, particularly in ters of intestinal infections. The methods of the control over the intestinal infections, as well wa the methods of diagnosis and treatment are still not able to create conditions for stable reduction of deseases. The nature of infectious deseases as well as their epidemiological patterns has been changed. Andryan A.A.2006]. Intestinal infections are also relevant in terms of economic losses.

Only rapid and completely development of the large-scale informational material about the infections, using informational technologies and integrated analysis epidemiological process give the opportunity to make probable previsions. With the help of such systematic, planned in advance effective fight against the infections and implementations of the preventive means in the field of epidemiology and in the case of the purposeful mode of work, it can be possible to reach noticeable results [Andryan A.A.2006].

Harmony increase of morbidity and the decreasing curve drawn with the communal improvement and planned order on the background of the preventive measures have rythmic frequencyover the time.The main factors of the frequency of epidemiological process are climatic, socio-economic, medicalgeographical impacts on the background of their complex influence, the analysis of the separate biorythms of the nozologies of infectious deseases makes it possible to identify epidemiological patterns, which are the basis of predictions. Periodical activation of deseases depends on the periodicity of infectional deseases, which is impossible to prevent with the help of antiepidemical measures, it is possible only to ease its course and to reduce theactivation /flare/ amplitude, to decrease epidemiological complications [Kartsev A.D.-2000].

Revelation of the periodicity and the anpalysis of the peaks in frequency will help us to make possible previssions and insert goal-orientation into the epidemiological control [Serebrennikov M.G., Pervozvanskiy A.A.-1965].

The periodicity of intestinal infections has been studied by many foreign researchers, but different authers represent only various stages of the morbidity variation of certain infections because of the lack of a unified approach to the definition of the periods and statistical characteristics [Vasilov V.I., Novikov A.I., Zamotin B.A.-1982].

It is necessary to take into account all the external factors influencing the epidemiological regularities and in the case of their durable influence epidemiological periodicity is stabilyzed. But in our era of scientific and technical progress, these factors are very variable, which creates a need to take into account changing regularities and the role of the factors influencing them on the deviations of the infections' periodicity. Therefore it is necessary to make profound, constant analysis to control the process of changing regularities and to make accurate predictions [Maksimov A.A.-1984].

The identification of the real frequency of the epidemiological process requires an unified approach to the problem, using modern informational technologies and mathematical methodology /Andryan A.A., Andryan V.E.-2007/.

\section{Material and Methods:}

Exact frequency and epidemical patterns cannot be revealed within the short-term study. How big is the observed period, the frequency phase- $T$ will be determined with greater accuracy. Taking into consideration etiological, clinical and epidemiological features of intestinal infections during the constal analysis of intestinal infection deseases (1977-2011) over the separate infections of intestinal group [Andryan A.A.-2007].

We use the method of spectral analysis of the group of harmony and time (variation) for the study of periodicity of any rythmic process [Anderson T.1977].

The process of mathematical modelization analysis is a final cost value of a real consonance (1).

$$
\begin{aligned}
& \mathrm{Z}_{(\mathrm{t})}=\mathrm{A}_{0}+\sum_{i=1}^{n}(\mathrm{Aj} \cos \omega \mathrm{jt}+\mathrm{Bj} \operatorname{sjn} \omega \mathrm{jt})+\Sigma_{(\mathrm{t})} \mathrm{t} \\
& =1, \mathrm{~N}
\end{aligned}
$$

Frequency of an epidemiological process is revealed by the analyzing method of harmony. The line of numbers derived from harmony method was used for prevision of epidemiological process, but it cannot be exact, as the method is correct for harmonic variations, when the amplitude and frequency are constant over the time. 
ANDRYAN A.A. / REVELATION OF ACUTE INTESTINAL INFEKTIONS' PERIODIC BIORYTHMS AND IDENTIFICATION OF DHE QUOLITATIVE PREVISIONS IN GYUMRI

In this way only the presence of the desease's periodicity is proved. The epidemiological frequency cannot be harmonic, as being a natural biological process, it is submitted to the influence of various changing external factors. That's why the harmonic analysis method gives incomplete predictions and disappointed many professionals to continue and extend the works to that direction.

We have used the spectral analysis method of the time-series to clarify real epidemiological pattern and to make predictions [Grendjer K., Khatanaka M.-1972], and with the help of Byui-Balo's hidden frequency's revelation method we have redused the probability of a possible error bringing the resulted frequency close to the real, natural biorythm.

For the spectral analysis of time series we need the spectral density function's value - $f(\omega \mathrm{j})$, which is the following:

$\mathrm{f}(\omega \mathrm{j})=1 / 2 \pi\left(\gamma_{0} \lambda_{0}+2 \sum_{k=1}^{m} \lambda_{\mathrm{k}} \gamma_{\mathrm{k}} \cos \omega \mathrm{j} \mathrm{k}\right)$

where $\gamma$-is the coveration value, $\lambda_{\mathbf{k}}$ is Parzen's coefficient (factor), which is received by the following formula (3):

$\lambda_{\mathbf{k}}={ }_{11-6 \mathrm{k}^{2}} / \mathrm{m}^{2}(1-\mathrm{k} / \mathrm{m}), 0 \leq \mathrm{k} \leq \mathrm{m} / 2$ (3)

$$
2(1-\mathrm{k} / \mathrm{m})^{3}, \mathrm{~m} / 2 \leq \mathbf{k} \leq \mathbf{m}
$$

In (2) and (3) expressions $m$ is the amount of of the points, with the help of which the spectre is evaluated.

The analysis of spectral dencity function is made with the help of the homonimic schedule, on, which there are peaks, which correspond to the most essential periods of the process. Moreover, if the peak is observed on the J point of the abscises, then it corresponds to the cycle with $t$ period where $\mathrm{T}=$ $2 \mathrm{~m} / \mathrm{j}$. Byui-Balo's method is identical with consonant analysis and differs only in the variability of the consonances'number during the observation. As in spectral analysis, so here spectral graphical time periods are made where peaks coincide with the real sycles of the observed process and are received in the case of the coincidence of experimental and real phases.

Spectral analysis of time series /time group/ have been made in the case of $m=12$ and $m=9$ where $m$ expresses the number of peaks by which the spectre is evaluated. During the following researches it was confirmed that comparatively reliable estimation is received in the case of $m=12$.

Only rapid and complete development of voluminous material and multiform analysis of the epidemiological process give the opportunity to make provisions. With the help of such systemized and productive fight planned beforehand, it's possible to get noticeable results. The works, made by us with the help of GIS / Geographic Informational System/ program and the above mentioned method in Gumry, give practical perceptible changes during the last 5 years. GIS geographical analysing system gives the opportunity to create voluminous numerical base and to use the program algorythms to make epidemiological analysis, to reveal regularities and to make previsions on their basis. [Jenkins G. Vatts D.-1971].

\section{Rezults and Discussion:}

Spectral analysisyears of time series, which characterize the avute intestinal infections, overoll morbidity of 34 years allows us to conform (establish) multirythmical characteristics of epidemiological Dynamics. It turns out, that there is a specific periodicity for the deseases of intestinal group with separate nozological gorms.

Thus, theoretically, with the help of the above mentioned method, the following periodicity has been received for the total group of intestinal deseases with - $5.9-6.0$ - 5 - 4,6 - 2,2 - 5,1 - 2,1 2,3 -year interval, which coincides with the practice activities with - 6 - 6 - 5 - 5 - 2 - 5 - 2 - 2 - regularity [picture 1] 
ANDRYAN A.A. / REVELATION OF ACUTE INTESTINAL INFEKTIONS' PERIODIC BIORYTHMS AND IDENTIFICATION OF DHE QUOLITATIVE PREVISIONS IN GYUMRI

\section{"Deseases with general diarrhea infections 1977-2011" intence indicator}

Dynamics of general group of intestinal infections morbidity in Gyumri 1977-2011

Parallel regularity is observed for the difficulty of the desease's clynical process, in the case of short cycles (2-3 years) the properties of the germ (pathogency, virusfull, tocsicus, stability) are reflected weakly, in the case of long cycles (56years) they are becoming more aggresive.

There are high and flashing morbidity's peaks with the above mentioned periods in the graphic of the AII /acure intestinal infection/ spectral dencity function, moreover, convenning structure of the vacillation /wavering is expressed by 2 basic rythms -3 and 5 years, moreover, t 5 years cycle is more significant and the morbidity is on higher level. By the same methodology the following variation series have been obtained for the nozologies of intestinal infections. According to the calculation, for the typhoid it will be $(2,5+3,1)-5,6-3,1-5,2-3,1-2,8$ biorhythm, actually 3-3-6-3-5-3-2- midactivation periods were recorded, expressed in years and the regularity of active years /according to the calculation and passed epidemiological-historical information for typhoid will be - 5 - 5 - 3 - 5 - 5 - 3 (1977-1980-1983-1987-1989-1992-1997-2002),

(cognition - the mentioned years are epidemiologically active, accompanied by high morbidity bolds are emidemically flashing and strained years).

For other salmonelosis /gastroenteritis/ infections, according to calculation 3,7+2,5)-4,1-3,3-4,2$(3,5+1,9)-3,2-2,9-3,0-2,4$ - biorythms were received, actually recorded -6-4-3-4-5-3-3-3-2- midactivation periods, expressed in years, the regularity for the active years for other salmonelosis infections will be the following : -3 3-4-5-3-3-4-5 -(1977-1983-19871990-1994-1999-2002):
For bacterial dysentery infections according to calculation $\quad-1,8-4,1-2,8-(2,2+2,3)-(3,1+1,7)$ (2,4+2,3)-3,4-3,0-3,9-1,9- biorythm was received, actually -2-4-2-4-5-4-3-3-4-2- midactivation periods were recorded expressed in years, the regularity for the active years of shigellosis will be - 3-2-2-3-2-2- (1977- 1979-1983-1985-1989-1994-1998-20012004-2008-2010-).

For the gastroenterocolitis of known and unknown genealogy revealed $-(3,2+2,8)-3,3-3,6-3,4-3,5-$ $(2,7+2,6)-(3,4+3,2)-$ biorythm, actually $-6-3-4-3-4-$ 5-7- midactivation periods have been recorded expressed in years, the regularity for the active years of morbidity with gastroenterocolotis will be -3-3-33-(-1977-1983-1986-1990-1993-1994-1997-19992002-2009).

According to the calculation for the viruses with the infectious Hepatitis A - $(2,9+1,8)-2,0-3,2-3,4-$ $(2,1+1,9)-3,0-3,3-2,0-1,7-3,4-(2,5+2,6)-\quad$ biorhythm have been received, actually -4-2-3-3-4-3-3-2-2-35 - midactivation periods were recorded expressed in years, the regularity for the active years of infectious Hepatitis A will be - 2-3-3-2-3-3- (1977-1981-19831984-1986-1989-1993-1996-1999-2001-2003):

The investigated regularities predict approximate, probable periods /times/ of the risk. It is well known, that the periodic increase and decrease of the morbidity is different for the different germs of the infections of intestinal group. It also has different subspecies of the germ . For example, in Gumri, periodic increase of the morbidity with Zonne shigelosis is observed, primery up to 1993 , once in every 6 years, after 1993 once in 3-4 years for Flexner shigeloz - 5-7 years. The additional 
ANDRYAN A.A. / REVELATION OF ACUTE INTESTINAL INFEKTIONS' PERIODIC BIORYTHMS AND IDENTIFICATION OF DHE QUOLITATIVE PREVISIONS IN GYUMRI

frequency of shigelozis ' different biotypes develops with -4-2-2-4 years periodicity. Beginning with 1997 the shigelozis biorythm has been changed. Sh. Sonnae become active with $2-3$ and 12 years periodic rythm, moreover 12 year cycle is more expressive, and Sh.Flexeri with 5-8 years frequency: 5 years cycle is more expressive. Sh. Flexneri's activations repeat oneself 2,5 times more often which explane its predominance in the shigellas' group $/ 85 \% /$.or in the group of other salmonelosis infections -yhe spectral dencity in S. typhimurium subtype's graphic of frequency function has 3 and 9 years frequency expressed in 3 years cycle and 6 and 12 year biorhythm for S.enteritidis subtype, which explains the predominance of $\mathrm{S}$. typhimurium. It is explained by its stability against/to/ the antibiotics and disinfectant materials. Thus, mentioned facts shows, that there is frequency biorythm of infectious deseases in connection with the requirement of the germ as a biological creature, to maintain its type. Separate biorythms have been revealed for the separate shchatiper of the viruses' germs of the intestinal group.

It should be noted that increases (peaks) and falls have general tendency to decrease and stabilize for the all intestinal infections in terms of cumulative, namely there are on a lower level, comparate with the previous periodicity.

Nowadays, beginning with 2001 , the role of the nutritional factors of the germs' transmission activated, which create a real danger of outbreaks' emergence, caused by the nutritional factor. This is a biological process, regulating the biological balance of type, which is also promoted by socio-economical insufficient conditions, no proper control towards food security, natural disasters, political crisis etc.. Therefore the reduction of morbidity implies the beginning of the next rise.

Byui-Baloy's method corrects the calculation of the frequency: reveals hidden frequency, deviation of biorhythms, caused by the above mentioned external factors affecting the variations.

The factors and conditions contributing to the activation of the epidemiological process can be the acvivation indicators of the epidemiological process (indicator-criteria), which are different for diver rent areas. Proceeding from the average relative rate of deseases characteristic to the area, the situation can be assessed as tense, morbidity - flashing, if the recorded cases of the desease exceeds the upper limit of that index. Biological cycle also depends on climatic factors associated with the improvement of conditions for development of the germ. The most important of these factors are the average summer air temperature and the number of average annual precipitation. Biological cycle is also promoted by natural factors of the global nature - the Earth's natural magnetic field and Sun's activations [Pokrovskaya T.V.-1971/]

\section{Inference:}

Using spectral analysis of time series, risk periods of intestinal separate infections were determined for Gyumri, which will be if not flashing, then probably years of high morbidity. According to previsions made by us, the frequency of separate infections has the following schedule:

Typhoid: 2002-2005-2007-2015-2018-2020-20252028-2030- the regularity has been changed from 1997, from -5-5-3 becoming -5-3-2.

Other salmonellosis: (-1999-2002-2005-2008-2010)(2013-2016-2019-2021-2023)-(2026- the regularity has been changed from -3-3-4-5- becoming -3-3-32-.

Bacterial dysentery: (-1994-1998-2001-2004-20082010)-(2014-2017-2020-2024-2026- the regularity has been changed from 1997, from -3-2-2-3becoming -4-3-3-4-2-.

Gastroenterocolitis: 2002 -2009-2012-2016-20192023-2029-) the regularity has been changed from 1997- from -3-3-3-4- becoming -7-3-4-3-4-6-.

Infectious Hepatitis A. (-1996-1999-2001-2003)(2006-2009-2011-2013)-(2016-2019-2021- the regularity has been changed from -3-3-2-3-3-2becoming -3-3-2-2-.

Food toxic infection (- 2003-2005-2009-2011-20132017)-(2021-2023-2027- the regularity has been changed from since 1997 from -2-4-2-2-4becoming -2-4-4-.

The spectral density of food toxic infections has frequency of 2 and 4 years, at first with 2 years and since 1997 with 4 years cycle expression, moreover food poisoning of staphyilococus origin is characteristic of 2 years cycle, and food poisoning of salmonelosis origin is characteristic of 4 years cycles.

\section{Conclusion:}

Only permanent moniitoring and current and deep epidemiological research gives the opportunity to reveal distinct epidemiological regularities. Spectral analysis, done with the help of modern informational technologies and the informational 
ANDRYAN A.A. / REVELATION OF ACUTE INTESTINAL INFEKTIONS' PERIODIC BIORYTHMS AND IDENTIFICATION OF DHE QUOLITATIVE PREVISIONS IN GYUMRI

base, resulted from logging in time series will give the opportunity to make variation number-series and appropriate previsions didactic. Computer mapping programs, especially Arc View GIS geographical informational system gives such an opportunity. With the help of digital maps resulted from this program, it is possible to get regional and allocation of infections, prevalence of indicators. It's also possible to determine the extent of contamination, which gives the opportunity to make epidemic measures intentional and effective to save means and resources.

Taking into consideration the effective use of the mentioned methodology:

- It is suggested to use the method of spectral analysis of time-series and method of ByuiBalo to investigate frequency of inflectional deseases.

\section{References:}

[1] Andryan A.A., Andryan V.E.[Epidemiological control with the use of Arc View GIS computer program] [published in Armenian] Methodological recommendations approved by the Ministry of Health Erevan 2007, with 55 pages/.

[2] Andryan A.A. [Epidemiological morbidity and previsions of acure intestinal infections in Gyumri][published in Armenian] Armenia's medical review /volume XLVII, N2 Erevan 2007, pg. 94-100/.

[3] Andryan A.A. [Investigation of the epidemiological regularities and the evaluation of intestinal infections'occurrence] [published in Armenian] "Human health V National Scientific Collection /international participation. Erevan 2006, pg. 313-315.

[4] Vasilov V. I., Novikov A.I, Zamotin B.A.[ Manifestations of cyclicity in the epidemical process of dysentery] [published in in Russian] microbiology, epidemiology, imunobiology, Moscow 1982 N5, pg 26-30.

[5] Karcev A.D.[Cyclicity and prevision of morbidity with shigelosis in Russia].[published
- To reveal the development of the cycle of epidemical process by mathematical method as an objectivecharacteristics of previsions.

- To make deep epidemiological research of the region to reveal the medical geographical characteristics of the area, epidemiological regularities and external factors, which affects the frequency?

- To use Arc View GIS software package to make the analysis fast and flowless, and to make the picture didactic.

Russian]J.Microbiology, Moscow 2000, N1, pg. 57-60.

[6] Anderson T. [Statistic analysis of time series] [published in in Russian] Moscow 1977.

[7] Jenkins G., Vatts D. [Spectral analysis and its applications] [published in in Russian] 1971-1972, part 1,2.

[8] Maksimov A.A [Long-term fluctuations of the number of animals, its causes and prognosis] [published in in Russian] News - Birsk 1984.

[9] Pokrovskaya T,V. [ The influence of solar activity on Earth's biosphere and otmosphere]. [published in in Russian] Moscow 1971.

[10] Serebrennikov M.G., Pervozvanskiy A.A. [Expression of the hidden periodisitie] [published in in Russian] Moscow 1965.

[11]Grendjer K., Khatanaka M. [Spectral analysis of time series] [published in in Russian] Moskva 1972.

[12] Andryan A.A. "Morbidity of enteric infections in Gumri" /2-nd International Medical Congress of Armenia-2007. p.209. 